\title{
Area Estimation for Web Browsing Performance of Users by Applying Numerical Method
}

\author{
Sharad Gangele, PhD \\ Associate Professor \\ Dept. of Computer Science \\ R.K.D.F. University, Bhopal M.P., India
}

\author{
Ashish Dongre, $\mathrm{PhD}$ \\ Director Technical Education \\ Government of state of M.P. \\ M.P., India
}

\begin{abstract}
Modern web browsers allow web developers to create highly interactive websites which are highly user friendly. Web browser technology has come a long way since its inception although browser compatibility issues remain a concern. This generates problem for the users to choose one among different browsers for accessing information on a required subject. Analysis of browser Share problem was first undertaken by Shukla and Singhai (2011) and they succeed to derive the expression for browser sharing. In fact, this expression has probability based bounded area but definite integral could not solve the problem of estimation of bounded area. In this paper an attempt has been made to estimate total probability area lying under the curve. Mathematical modeling utilized the application of Simpson 3/8 rule in browser sharing phenomena. It is also established by the study that such bounded area possesses linear relationship with the browser failure probability.
\end{abstract}

\section{Keywords}

Browser, Simpson 3/8 rule, Browser failure probability, Area approximation

\section{INTRODUCTION}

Web browser is an application program that provides a way to look at and interact with all the information on the World Wide Web for fulfillment of many needs. Browser popularity in the market is also an important factor. Naldi(2002) discusses an application of Markov chain on traffic share scenario whereas Shukla and Singhai (2011) utilized this application on browser sharing prospect and some expression of browser share was derived when two browser are installed in a computer system. This expression of browser share gives an area. This bounded area of browser share is a variable therefore many result can be derived from it. Now the problem is how to estimate this area. In this paper a procedure has been suggested for estimating such an appropriate area by using Simpson 3/8 rule which is available in numerical analysis literature.

\section{REVIEW OF LITERATURE}

Newby and Dagg (2002) proposed inspection and maintenance for stochastically deteriorating systems for average cost criteria with the help of markov chain model. Agarwal and Kaur (2008) discuss a reliability analysis of fault-tolerant multistage interconnection networks and develop a methodology for it. Medhi (1991) has given detail discussion on random movement in every aspect of real time situation through markov chain. Naldi (2002) proposed a new framework on internet traffic share phenomena which is involve between two operator environments. Catledge and Pitkow (1995) suggested some characterizing browsing strategies in the World Wide Web in the field of computer networking. Shukla et al.(2007) advocate a model based analysis for space division switching and find some new result for it. Shukla et al.(2010) examine crime based user behaviour analysis in the setup of multi operator environment case. Naldi (1999) focused on measurement based modelling of internet dial-up access connections in a new way with the help of markov chain model. Shukla and Thakur (2009) performed state probability analysis of users in internet access traffic sharing in various competitive operator environment cases. Shukla et al.(2009) attempt for rest state analysis in internet traffic distribution in multi-operator environment situation. One more similar study is performed due to Shukla and Gadewar (2007) for stochastic model based analysis for cell movement in a knockout switching in the field of networking. Shukla and Singhai (2011) have a useful contribution on user's web browsing behaviour study by using Markov chain model. Shukla et al. (2011) conducted a study for elasticity examination of web-browsing behaviour of users with the help of first derivative of browser share expression. Shukla et al. (2012a,b,c) develop some new properties of traffic share phenomena in various heterogeneous computer network system through least square based curve fitting technique .Gangele et al.(2014a,b) have given a mathematical approach for area estimation of internet traffic share problem in two operators environment situation and develop new aspect for it. Gangele and dongre (2014c, d) have described index based analysis in two call based setup for the judgement of users behaviour in various network situations. Gangele (2014) analysed a new approach for area computation of traffic sharing through Simpson 1/3 rule used in numerical analysis. Shukla et al. (2015) advocate probability based approximation of the traffic sharing phenomena by using numerical analysis techniques between two operators in a computer network environment. Shuka and Singhai (2011) derived the following expression of browser sharing 
$\bar{B}_{1}=\left(1-b_{1}\right)\left(1-P_{C}\right)\left\{\frac{P+(1-P)\left(1-P_{q}\right) b_{2}}{1-b_{1} b_{2}\left(1-P_{q}\right)^{2}}\right\}$

The graph of above expression is based on browser failure probability $\left(\mathrm{b}_{1}\right.$ or $\left.\mathrm{b}_{2}\right)$ and browse sharing $\left(\bar{B}_{1}\right)$ of browser $\mathrm{B}_{1}$. It provides a bounded area $\mathrm{A}$ within curve. Basically this bounded area is a variable therefore many result can be drawn, if we estimate such a bounded area .Now the problem is how to estimate this area .In this paper we develop a method for estimating such a bounded area by applying Simpson's 3/8 method which is available in numerical analysis literature.

\section{SIMPSON'S 3/8 RULE}

Now let $\mathrm{y}=\mathrm{f}(\mathrm{x})$ be a function to be integrated in the range a to $\mathrm{b}(\mathrm{a}<\mathrm{b})$. Using functional relationship, we can write $\mathrm{n}$ different discrete values of $x$ in range $a-b$, and can write different $\mathrm{y}$ using $\mathrm{y}=\mathrm{f}(\mathrm{x})$ as below:

$$
\begin{aligned}
& x: \quad x_{0}, x_{1} \ldots x_{n} \\
& y: \quad y_{0}, y_{1} \ldots y_{n}, ; \quad(i=1,2,3,4, \ldots n) ;
\end{aligned}
$$

Where $\mathrm{a}=\mathrm{x}_{0}, \mathrm{x}_{1}<\mathrm{x}_{2}<\mathrm{x}_{3} \ldots<\mathrm{x}_{\mathrm{n}}=\mathrm{b}$ and differencing $\mathrm{h}=\left(\mathrm{x}_{\mathrm{i}+1}\right.$ $-\mathrm{x}_{\mathrm{i}}$ ) is like equal interval.

$$
\begin{aligned}
& I=\int_{b}^{a} f(x) d x=\int_{b}^{a} y d x \\
& =\frac{3 h}{8}\left[\begin{array}{c}
\left(y_{0}+y_{n}\right)+ \\
3\left(\begin{array}{l}
y_{1}+y_{2}+y_{4}+y_{5}+y_{7}+ \\
y_{8}+\ldots+y_{n-2}+y_{n-1}
\end{array}\right)+ \\
2\left(y_{3}+y_{6}+y_{9}+\ldots+y_{n-3}\right)
\end{array}\right]
\end{aligned}
$$

Which is known as Simpson's three eight rule of integration used in numerical analysis.

\section{APPLICATION OF SIMPSON'S 3/8 METHOD}

We take the followings and consider $B_{1}=f\left(b_{1}\right)$ as a function and assume $\mathrm{X}=$ Browser failure probability $\left(\mathrm{b}_{1}\right), \mathrm{Y}=$ Browser sharing is equal to $\bar{B}_{1}$ and want to evaluate the following integral (as discussed by Shuka and Singhai (2011)) in the limit 0 to 1 are constraints:

$$
\begin{aligned}
& I=\int_{0}^{1} f\left(b_{1}\right) d b_{1}= \\
& \int_{0}^{1}\left(1-b_{1}\right)\left(1-P_{C}\right)\left\{\frac{P+(1-P)\left(1-P_{q}\right) b_{2}}{1-b_{1} b_{2}\left(1-P_{q}\right)^{2}}\right\} d b_{1}
\end{aligned}
$$

\begin{tabular}{|c|c|c|c|c|c|c|c|c|c|}
\hline \multicolumn{10}{|c|}{ TABLE 1-[ For Figure (a) Where $\left.\left(\mathbf{P}=\mathbf{0 . 3 5}, \mathbf{P}_{\mathbf{q}}=\mathbf{0 . 2 0}, \mathbf{p}_{\mathbf{c}}=\mathbf{0 . 1 5}, \mathbf{h = 0 . 0 5}\right)\right]$} \\
\hline $\mathbf{b}_{\mathbf{2}}$ & $\mathbf{0 . 1}$ & $\mathbf{0 . 2}$ & $\mathbf{0 . 3}$ & $\mathbf{0 . 4}$ & $\mathbf{0 . 5}$ & $\mathbf{0 . 6}$ & $\mathbf{0 . 7}$ & $\mathbf{0 . 8}$ & $\mathbf{0 . 9}$ \\
\hline $\mathbf{b}_{\mathbf{1}}$ & $\overline{\boldsymbol{B}}_{1}$ & $\overline{\boldsymbol{B}}_{1}$ & $\overline{\boldsymbol{B}}_{1}$ & $\overline{\boldsymbol{B}}_{1}$ & $\overline{\boldsymbol{B}}_{1}$ & $\overline{\boldsymbol{B}}_{1}$ & $\bar{B}_{1}$ & $\bar{B}_{1}$ & $\bar{B}_{1}$ \\
\hline $\mathbf{0}$ & 0.3417 & 0.3859 & 0.4301 & 0.4743 & 0.5185 & 0.5627 & 0.6069 & 0.6511 & 0.6953 \\
\hline $\mathbf{0 . 0 5}$ & 0.3257 & 0.369 & 0.4126 & 0.4564 & 0.5006 & 0.545 & 0.5898 & 0.6348 & 0.6801 \\
\hline $\mathbf{0 . 1}$ & 0.3095 & 0.3518 & 0.3947 & 0.4381 & 0.4821 & 0.5267 & 0.5718 & 0.6176 & 0.664 \\
\hline $\mathbf{0 . 1 5}$ & 0.2933 & 0.3344 & 0.3764 & 0.4193 & 0.4629 & 0.5075 & 0.553 & 0.5995 & 0.6469 \\
\hline $\mathbf{0 . 2}$ & 0.2769 & 0.3168 & 0.3578 & 0.3999 & 0.4432 & 0.4876 & 0.5333 & 0.5803 & 0.6287 \\
\hline $\mathbf{0 . 2 5}$ & 0.2604 & 0.299 & 0.3388 & 0.3800 & 0.4227 & 0.4668 & 0.5126 & 0.5600 & 0.6092 \\
\hline $\mathbf{0 . 3}$ & 0.2439 & 0.2809 & 0.3195 & 0.3596 & 0.4015 & 0.4452 & 0.4908 & 0.5385 & 0.5884 \\
\hline $\mathbf{0 . 3 5}$ & 0.2272 & 0.2626 & 0.2997 & 0.3386 & 0.3795 & 0.4225 & 0.4678 & 0.5156 & 0.5661 \\
\hline
\end{tabular}




\begin{tabular}{|c|c|c|c|c|c|c|c|c|c|}
\hline $\mathbf{0 . 4}$ & 0.2104 & 0.244 & 0.2795 & 0.3170 & 0.3568 & 0.3989 & 0.4436 & 0.4913 & 0.5421 \\
\hline $\mathbf{0 . 4 5}$ & 0.1935 & 0.2252 & 0.2589 & 0.2948 & 0.3331 & 0.3741 & 0.4181 & 0.4653 & 0.5162 \\
\hline $\mathbf{0 . 5}$ & 0.1765 & 0.2061 & 0.2379 & 0.272 & 0.3086 & 0.3482 & 0.391 & 0.4376 & 0.4883 \\
\hline $\mathbf{0 . 5 5}$ & 0.1594 & 0.1868 & 0.2164 & 0.2484 & 0.2832 & 0.321 & 0.3624 & 0.4078 & 0.458 \\
\hline $\mathbf{0 . 6}$ & 0.1421 & 0.1672 & 0.1944 & 0.2241 & 0.2567 & 0.2925 & 0.332 & 0.3759 & 0.425 \\
\hline $\mathbf{0 . 6 5}$ & 0.1248 & 0.1473 & 0.172 & 0.1991 & 0.2291 & 0.2625 & 0.2997 & 0.3416 & 0.389 \\
\hline $\mathbf{0 . 7}$ & 0.1073 & 0.1272 & 0.1491 & 0.1734 & 0.2005 & 0.2309 & 0.2653 & 0.3044 & 0.3495 \\
\hline $\mathbf{0 . 7 5}$ & 0.0897 & 0.1067 & 0.1256 & 0.1468 & 0.1706 & 0.1976 & 0.2285 & 0.2642 & 0.306 \\
\hline $\mathbf{0 . 8}$ & 0.072 & 0.086 & 0.1016 & 0.1193 & 0.1394 & 0.1624 & 0.1892 & 0.2206 & 0.2579 \\
\hline $\mathbf{0 . 8 5}$ & 0.0542 & 0.065 & 0.0771 & 0.0909 & 0.1068 & 0.1253 & 0.147 & 0.1729 & 0.2043 \\
\hline $\mathbf{0 . 9}$ & 0.0363 & 0.0436 & 0.052 & 0.0616 & 0.0728 & 0.086 & 0.1017 & 0.1208 & 0.1444 \\
\hline $\mathbf{0 . 9 5}$ & 0.0182 & 0.022 & 0.0263 & 0.0313 & 0.0372 & 0.0443 & 0.0528 & 0.0634 & 0.0768 \\
\hline AREA(A)= & $\mathbf{0 . 1 7 3 8}$ & $\mathbf{0 . 2 0 0 8}$ & $\mathbf{0 . 2 2 9 2}$ & $\mathbf{0 . 2 5 9 1}$ & $\mathbf{0 . 2 9 0 7 8}$ & $\mathbf{0 . 3 2 4 5}$ & $\mathbf{0 . 3 6 0 5}$ & $\mathbf{0 . 3 9 9 3}$ & $\mathbf{0 . 4 4 1 3}$ \\
\hline
\end{tabular}

Looking over table 1 area depends on browser failure probability $b_{2}$. For higher value of $b_{2}$ area is high where as for lower value it low. The growth rate is from $17 \%$ to $44 \%$ at maximum increment of browser failure probability $b_{2}$ and for some constant parameter $\mathrm{P}=35 \%, \mathrm{P}_{\mathrm{q}}=20 \%$ and $\mathrm{p}_{\mathrm{c}}=15 \%$.

TABLE 2-[ For Figure (b) Where $\left.\left(\mathrm{b}_{2}=0.45, \mathrm{P}_{\mathrm{q}}=\mathbf{0 . 2 5}, \mathrm{p}_{\mathrm{c}}=\mathbf{0 . 3}, \mathrm{h}=\mathbf{0 . 0 5}\right)\right]$

\begin{tabular}{|c|c|c|c|c|c|c|c|c|c|}
\hline $\mathbf{P}$ & 0.1 & 0.2 & 0.3 & 0.4 & 0.5 & 0.6 & 0.7 & 0.8 & 0.9 \\
\hline $\mathbf{b}_{1}$ & $B_{1}$ & $B_{1}$ & $B_{1}$ & $B_{1}$ & $B_{1}$ & $B_{1}$ & $B_{1}$ & $B_{1}$ & $B_{1}$ \\
\hline 0 & 0.2826 & 0.329 & 0.3754 & 0.4218 & 0.4681 & 0.5145 & 0.5609 & 0.6073 & 0.6536 \\
\hline 0.05 & 0.2719 & 0.3166 & 0.3612 & 0.4058 & 0.4504 & 0.495 & 0.5397 & 0.5843 & 0.6289 \\
\hline 0.1 & 0.261 & 0.3038 & 0.3466 & 0.3894 & 0.4323 & 0.4751 & 0.5179 & 0.5607 & 0.6035 \\
\hline 0.15 & 0.2497 & 0.2907 & 0.3317 & 0.3726 & 0.4136 & 0.4546 & 0.4956 & 0.5365 & 0.5775 \\
\hline 0.2 & 0.2382 & 0.2772 & 0.3163 & 0.3554 & 0.3945 & 0.4335 & 0.4726 & 0.5117 & 0.5508 \\
\hline 0.25 & 0.2263 & 0.2634 & 0.3006 & 0.3377 & 0.3748 & 0.4119 & 0.4491 & 0.4862 & 0.5233 \\
\hline 0.3 & 0.2141 & 0.2492 & 0.2844 & 0.3195 & 0.3546 & 0.3897 & 0.4249 & 0.4600 & 0.4951 \\
\hline 0.35 & 0.2016 & 0.2346 & 0.2677 & 0.3008 & 0.3339 & 0.3669 & 0.4000 & 0.4331 & 0.4662 \\
\hline 0.4 & 0.1887 & 0.2196 & 0.2506 & 0.2816 & 0.3125 & 0.3435 & 0.3744 & 0.4054 & 0.4364 \\
\hline 0.45 & 0.1754 & 0.2042 & 0.2330 & 0.2618 & 0.2906 & 0.3194 & 0.3481 & 0.3769 & 0.4057 \\
\hline 0.5 & 0.1618 & 0.1883 & 0.2149 & 0.2414 & 0.268 & 0.2945 & 0.3211 & 0.3476 & 0.3742 \\
\hline 0.55 & 0.1478 & 0.172 & 0.1962 & 0.2205 & 0.2447 & 0.2690 & 0.2932 & 0.3175 & 0.3417 \\
\hline 0.6 & 0.1333 & 0.1552 & 0.177 & 0.1989 & 0.2208 & 0.2427 & 0.2645 & 0.2864 & 0.3083 \\
\hline 0.65 & 0.1184 & 0.1378 & 0.1573 & 0.1767 & 0.1961 & 0.2155 & 0.2350 & 0.2544 & 0.2738 \\
\hline 0.7 & 0.103 & 0.12 & 0.1369 & 0.1538 & 0.1707 & 0.1876 & 0.2045 & 0.2214 & 0.2383 \\
\hline 0.75 & 0.0872 & 0.1015 & 0.1158 & 0.1301 & 0.1445 & 0.1588 & 0.1731 & 0.1874 & 0.2017 \\
\hline 0.8 & 0.0709 & 0.0825 & 0.0941 & 0.1058 & 0.1174 & 0.129 & 0.1407 & 0.1523 & 0.1639 \\
\hline 0.85 & 0.054 & 0.0629 & 0.0717 & 0.0806 & 0.0895 & 0.0983 & 0.1072 & 0.1161 & 0.1249 \\
\hline 0.9 & 0.0366 & 0.0426 & 0.0486 & 0.0546 & 0.0606 & 0.0666 & 0.0726 & 0.0786 & 0.0846 \\
\hline 0.95 & 0.0186 & 0.0217 & 0.0247 & 0.0278 & 0.0308 & 0.0339 & 0.0369 & 0.0400 & 0.043 \\
\hline $\operatorname{AREA}(\mathbf{A})=$ & 0.1542 & 0.1795 & 0.2048 & 0.2301 & 0.2554 & 0.2807 & 0.306 & 0.3313 & 0.3566 \\
\hline
\end{tabular}


The table 2 shows the fact that for variation of $\mathrm{P}$ bounded area increases when browser failure probability $\mathrm{b}_{2}$ is $45 \%, \mathrm{P}_{\mathrm{q}}$ is

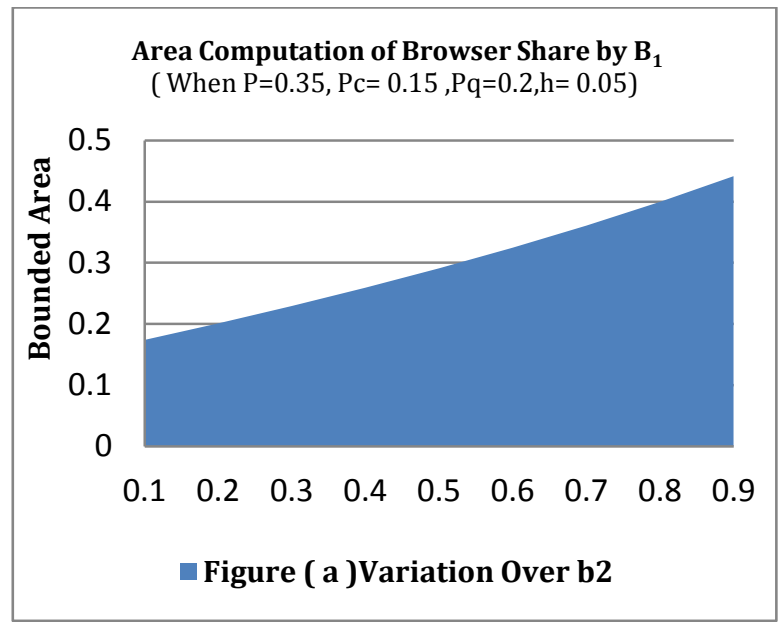

Figure(a) support the fact of table 1 for increasing pattern over browser failure probability $b_{2}$ maximum bounded area is nearly $45 \%$ with some constant parameter where as figure (b)
$25 \%$ and quitting probability $\mathrm{p}_{\mathrm{c}}$ is $30 \%$ with some little increment of browser failure $b_{1}$ by $5 \%$.

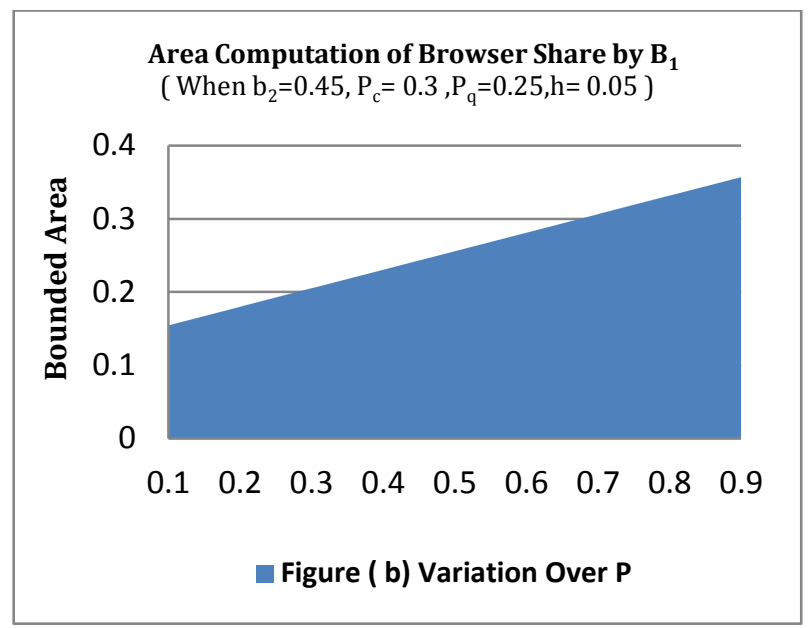

justify the fact related to table 2 that maximum limit of area is $35 \%$ with increase pattern for variation over $p$.

\begin{tabular}{|c|c|c|c|c|c|c|c|c|c|}
\hline \multicolumn{10}{|c|}{ TABLE 3-[ For Figure $(c)$ Where $\left.\left(b_{2}=0.05, P=0.25, p_{q}=0.45, h=0.05\right)\right]$} \\
\hline Pc & 0.1 & 0.2 & 0.3 & 0.4 & 0.5 & 0.6 & 0.7 & 0.8 & 0.9 \\
\hline$b_{1}$ & $\bar{B}_{1}$ & $\bar{B}_{1}$ & $\bar{B}_{1}$ & $\bar{B}_{1}$ & $\bar{B}_{1}$ & $\bar{B}_{1}$ & $\bar{B}_{1}$ & $\bar{B}_{1}$ & $\bar{B}_{1}$ \\
\hline O & 0.2436 & 0.2165 & 0.1894 & 0.1624 & 0.1353 & 0.1083 & 0.0812 & 0.0541 & 0.0271 \\
\hline 0.05 & 0.2316 & 0.2058 & 0.1801 & 0.1544 & 0.1286 & 0.1029 & 0.0772 & 0.0515 & 0.0257 \\
\hline 0.1 & 0.2195 & 0.1951 & 0.1708 & 0.1464 & 0.122 & 0.0976 & 0.0732 & 0.0488 & 0.0244 \\
\hline 0.15 & 0.2075 & 0.1844 & 0.1614 & 0.1383 & 0.1153 & 0.0922 & 0.0692 & 0.0461 & 0.0231 \\
\hline 0.2 & 0.1954 & 0.1737 & 0.152 & 0.1303 & 0.1086 & 0.0869 & 0.0651 & 0.0434 & 0.0217 \\
\hline 0.25 & 0.1834 & 0.163 & 0.1426 & 0.1222 & 0.1019 & 0.0815 & 0.0611 & 0.0407 & 0.0204 \\
\hline 0.3 & 0.1713 & 0.1522 & 0.1332 & 0.1142 & 0.0952 & 0.0761 & 0.0571 & 0.0381 & 0.019 \\
\hline 0.35 & 0.1592 & 0.1415 & 0.1238 & 0.1061 & 0.0884 & 0.0707 & 0.0531 & 0.0354 & 0.0177 \\
\hline 0.4 & 0.147 & 0.1307 & 0.1144 & 0.098 & 0.0817 & 0.0653 & 0.049 & 0.0327 & 0.0163 \\
\hline 0.45 & 0.1349 & 0.1199 & 0.1049 & 0.0899 & 0.0749 & 0.0599 & 0.0450 & 0.0300 & 0.015 \\
\hline 0.5 & 0.1227 & 0.1091 & 0.0954 & 0.0818 & 0.0682 & 0.0545 & 0.0409 & 0.0273 & 0.0136 \\
\hline 0.55 & 0.1105 & 0.0982 & 0.086 & 0.0737 & 0.0614 & 0.0491 & 0.0368 & 0.0246 & 0.0123 \\
\hline 0.6 & 0.0983 & 0.0874 & 0.0765 & 0.0655 & 0.0546 & 0.0437 & 0.0328 & 0.0218 & 0.0109 \\
\hline 0.65 & 0.0861 & 0.0765 & 0.067 & 0.0574 & 0.0478 & 0.0383 & 0.0287 & 0.0191 & 0.0096 \\
\hline 0.7 & 0.0739 & 0.0656 & 0.0574 & 0.0492 & 0.041 & 0.0328 & 0.0246 & 0.0164 & 0.0082 \\
\hline 0.75 & 0.0616 & 0.0547 & 0.0479 & 0.0411 & 0.0342 & 0.0274 & 0.0205 & 0.0137 & 0.0068 \\
\hline 0.8 & 0.0493 & 0.0438 & 0.0384 & 0.0329 & 0.0274 & 0.0219 & 0.0164 & 0.011 & 0.0055 \\
\hline 0.85 & 0.037 & 0.0329 & 0.0288 & 0.0247 & 0.0206 & 0.0164 & 0.0123 & 0.0082 & 0.0041 \\
\hline 0.9 & 0.0247 & 0.0219 & 0.0192 & 0.0165 & 0.0137 & 0.011 & 0.0082 & 0.0055 & 0.0027 \\
\hline 0.95 & 0.0124 & 0.011 & 0.0096 & 0.0082 & 0.0069 & 0.0055 & 0.0041 & 0.0027 & 0.0014 \\
\hline $\operatorname{AREA}(A)=$ & 0.1219 & 0.1083 & 0.0948 & 0.0812 & 0.0677 & 0.0542 & 0.0406 & 0.0271 & 0.0135 \\
\hline
\end{tabular}

Table 3 indicate the fact that for some constant parameter when browser failure probability $\mathrm{b}_{2}$ is $5 \%, \mathrm{P}$ is $25 \%$ and quitting probability $\mathrm{p}_{\mathrm{q}}$ is $45 \%$ area is downward trend for varying parameter $\mathrm{P}_{\mathrm{c}}$ by $10 \%$. 


\begin{tabular}{|c|c|c|c|c|c|c|c|c|c|}
\hline \multicolumn{10}{|c|}{ TABLE 4-[ For Figure $(d)$ Where $\left.\left(b_{2}=0.35, P=0.45, P_{c}=0.15, h=0.05\right)\right]$} \\
\hline $\mathbf{P}_{\mathbf{q}}$ & 0.1 & 0.2 & 0.3 & 0.4 & 0.5 & 0.6 & 0.7 & 0.8 & 0.9 \\
\hline $\mathbf{b}_{1}$ & $\bar{B}_{1}$ & $\bar{B}_{1}$ & $\bar{B}_{1}$ & $\bar{B}_{1}$ & $\bar{B}_{1}$ & $\bar{B}_{1}$ & $\bar{B}_{1}$ & $\bar{B}_{1}$ & $\bar{B}_{1}$ \\
\hline $\mathbf{0}$ & 0.5298 & 0.5134 & 0.497 & 0.4807 & 0.4643 & 0.448 & 0.4316 & 0.4152 & 0.3989 \\
\hline 0.05 & 0.5105 & 0.4933 & 0.4763 & 0.4595 & 0.443 & 0.4267 & 0.4107 & 0.3947 & 0.3790 \\
\hline 0.1 & 0.4907 & 0.4726 & 0.4551 & 0.4381 & 0.4216 & 0.4054 & 0.3897 & 0.3742 & 0.3591 \\
\hline 0.15 & 0.4703 & 0.4516 & 0.4336 & 0.4164 & 0.3999 & 0.384 & 0.3686 & 0.3537 & 0.3392 \\
\hline 0.2 & 0.4493 & 0.43 & 0.4118 & 0.3945 & 0.3781 & 0.3624 & 0.3475 & 0.3331 & 0.3193 \\
\hline 0.25 & 0.4276 & 0.4079 & 0.3895 & 0.3722 & 0.356 & 0.3407 & 0.3263 & 0.3125 & 0.2994 \\
\hline 0.3 & 0.4053 & 0.3853 & 0.3668 & 0.3497 & 0.3338 & 0.3189 & 0.305 & 0.2919 & 0.2795 \\
\hline 0.35 & 0.3823 & 0.3621 & 0.3437 & 0.3269 & 0.3113 & 0.297 & 0.2837 & 0.2712 & 0.2596 \\
\hline 0.4 & 0.3585 & 0.3384 & 0.3202 & 0.3037 & 0.2887 & 0.2749 & 0.2623 & 0.2505 & 0.2397 \\
\hline 0.45 & 0.334 & 0.314 & 0.2962 & 0.2803 & 0.2658 & 0.2527 & 0.2408 & 0.2298 & 0.2197 \\
\hline 0.5 & 0.3086 & 0.2891 & 0.2718 & 0.2565 & 0.2428 & 0.2304 & 0.2192 & 0.2091 & 0.1998 \\
\hline 0.55 & 0.2824 & 0.2635 & 0.247 & 0.2324 & 0.2195 & 0.208 & 0.1976 & 0.1883 & 0.1798 \\
\hline 0.6 & 0.2553 & 0.2372 & 0.2216 & 0.2080 & 0.196 & 0.1854 & 0.176 & 0.1675 & 0.1599 \\
\hline 0.65 & 0.2273 & 0.2103 & 0.1958 & 0.1832 & 0.1723 & 0.1627 & 0.1542 & 0.1467 & 0.1399 \\
\hline 0.7 & 0.1983 & 0.1827 & 0.1695 & 0.1582 & 0.1484 & 0.1399 & 0.1324 & 0.1258 & 0.1200 \\
\hline 0.75 & 0.1682 & 0.1543 & 0.1426 & 0.1327 & 0.1242 & 0.1169 & 0.1105 & 0.1049 & 0.1000 \\
\hline 0.8 & 0.137 & 0.1251 & 0.1152 & 0.1069 & 0.0999 & 0.0938 & 0.0885 & 0.084 & 0.0800 \\
\hline 0.85 & 0.1047 & 0.0951 & 0.0873 & 0.0807 & 0.0752 & 0.0706 & 0.0665 & 0.063 & 0.0600 \\
\hline 0.9 & 0.0711 & 0.0643 & 0.0588 & 0.0542 & 0.0504 & 0.0472 & 0.0444 & 0.0421 & 0.0400 \\
\hline 0.95 & 0.0363 & 0.0326 & 0.0297 & 0.0273 & 0.0253 & 0.0237 & 0.0222 & 0.021 & 0.0200 \\
\hline $\operatorname{AREA}(\mathbf{A})=$ & 0.2926 & 0.2769 & 0.2628 & 0.2499 & 0.2381 & 0.2272 & 0.2171 & 0.2077 & 0.1988 \\
\hline
\end{tabular}

Table 4 depicts decrement pattern of bounded area with the variation of web browser parameter $\mathrm{b}_{2}$ by $35 \%, \mathrm{P}$ is $45 \%$ and $\mathrm{p}_{\mathrm{c}}$ is $15 \%$ with some little increment of parameter $\mathrm{p}_{\mathrm{q}}$ by $10 \%$.

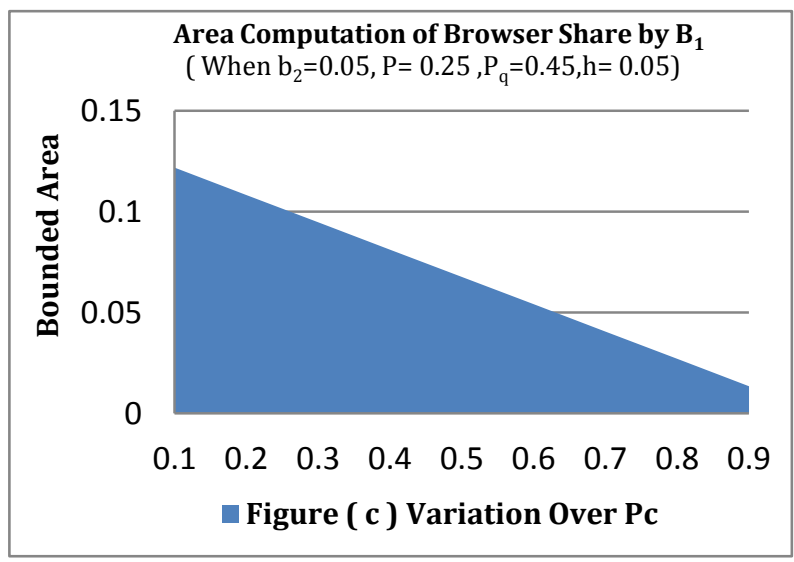

Figure (d) support the fact related to table no. 4 that a linear downward trend was seen at different browser sharing
Figure (c) related to table 3 depicts a downward trend between quitting probability $\mathrm{P}_{\mathrm{c}}$ and bounded area (A) for some fixed parameter and nearly $12 \%$ maximum area was seen.

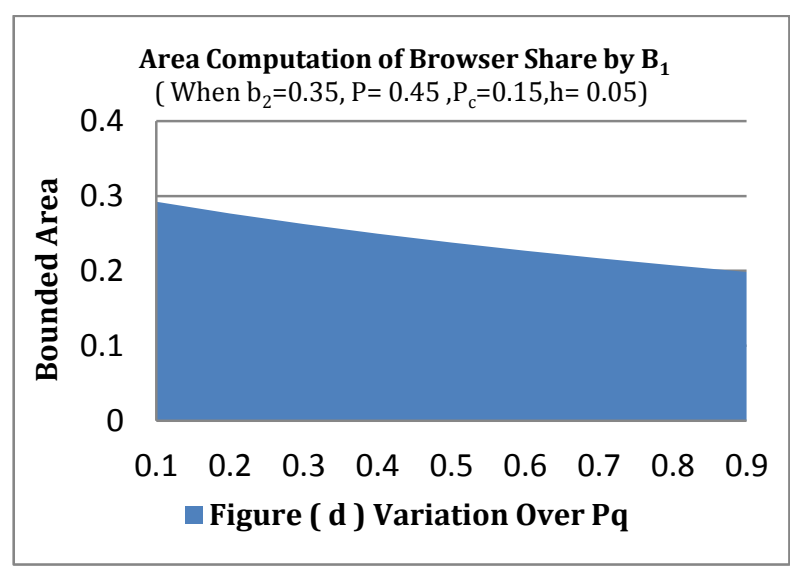

parameter .Nearly $28 \%$ maximum area was found when quitting probability is $15 \%$. 


\section{CONCLUSION}

It is evident from the study that browser share is directly associated with browser failure probability, quitting probability and other parameters. Browser sharing varies from time to time and region to region .Largest achievable area is $45 \%$ when $\mathrm{P}=35 \%, \mathrm{P}_{\mathrm{c}}=15 \%$ and $\mathrm{P}_{\mathrm{q}}=20 \%$ .Moreover study reveals linear relationship between bounded area and browser failure probability. These parameters affect browser share status at different stages of browser failure. Simpson 3/8 method is quite effective for estimating area of browse share in a setup of two browser environment.

\section{REFERENCES}

[1] M. Newby and R. Dagg (2002): Optical inspection and maintenance for stochastically deteriorating systems, average cost criteria, Jour. Ind. Stat. Asso., Vol. 40, Issue No. 2, pp. 169-198.

[2] Agarwal, Rinkle and Kaur, Lakhwinder (2008): On reliability analysis of fault-tolerant multistage interconnection networks, International Journal of Computer Science and Security (IJCSS) Vol. 02, Issue No. 04, pp. 1-8.

[3] Medhi, J.(1991): Stochastic Processes, Wiley Eastern Limited (Fourth reprint), New Delhi.

[4] Naldi, M. (2002): Internet access traffic sharing in a Multi-operator environment, Computer Networks, Vol. 38, Issue No. 06, pp.809-824.

[5] Catledge, L. D. and Pitkow J. E. (1995):Characterizing browsing strategies in the World Wide Web, Computer Networks and ISDN Systems, 26 (6),pp 1065.

[6] Shukla, D., Gadewar, S. and Pathak, R.K. (2007): A stochastic model for space division switches in computer networks, International Journal of Applied Mathematics and Computation, Elsevier Journals, Vol. 184, Issue No. 02, pp235-269.

[7] Tiwari, Virendra, Thakur, Sanjay and Shukla, D.(2010):Cyber crime analysis for multi-dimensional effect in computer network, Journal of Global Research in Computer Science, Vol.1, No. 4., pp 3137.

[8] M. Naldi (1999): Measurement based modelling of Internet Dial-up Access Connections, Computer Networks, Vol. 31, Issue 22, pp. 2381-2390.

[9] Shukla, D., Thakur, Sanjay (2009): State Probability Analysis of Users in Internet between two Operators, International Journal of Advanced Networking and Applications (IJANA), Vol. 1, Issue 2, pp. 90-95.

[10] Shukla, D., Tiwari, M.,Thakur, S. Tiwari,V.( 2009): Rest state analysis in internet traffic distribution in multi-operator environment, Research Journal of Management and Information Technology (GNIM's), Vol 1, Issue 1, pp. 72-82.

[11] Shukla, D.,Gadewar, Surendra (2007):Stochastic model for cell movement in a knockout switch in computer networks, Journal of High Speed Network, Vol.16, No.3, pp. 310-332.

[12] Shukla, D.,Gadewar, S. Pathak, R.K.,(2007): A Stochastic Model for Space-Division Switches in Computer Networks, Applied Mathematics and
Computation (Elsevier Journal), Vol. 184, Issue 2, pp. 235-269.

[13] Shukla, D. and Singhai, Rahul (2011): Analysis of user web browsing behaviour using Markov chain model, International Journal of Advanced Networking and Application (IJANA), Vol. 2, No. 5, pp. 824-830.

[14] Shukla, D.,Gangele, Sharad, Singhai, R., Verma, Kapil (2011): Elasticity analysis of web-browsing behaviour of users, International Journal of Advanced Networking and Application(IJANA), Vol. 3, No.3, pp. 1162-1168.

[15] Shukla, D., Verma, Kapil and Gangele, Sharad, (2012a): Curve Fitting Approximation In Internet Traffic Distribution In Computer Network In Two Market Environment, International Journal of Computer Science and Information Security (IJCSIS), Vol. 10, Issue 05, pp. 71-78.

[16] Shukla, D., Verma, Kapil and Gangele, Sharad, (2012b): Least Square Fitting Applications under Rest State Environment in Internet Traffic Sharing in Computer Network, International Journal of Computer Science and Telecommunications (IJCST), Vol.3, Issue 05, pp.43-51

[17] Shukla, D., Verma, Kapil, Dubey, Jayant and Gangele, Sharad (2012c): Cyber Crime Based Curve Fitting Analysis in Internet Traffic Sharing in Computer Network, International Journal of Computer Application (IJCA), Vol.46 No.22, pp. 41-51.

[18] Gangele, S. Verma, K. and Shukla, D., (2014a): Bounded Area Estimation of Internet Traffic Share Curve, International Journal of Computer Science and Business Informatics, Vol. 10, No. 1, pp. 54-67.

[19] Gangele,Sharad,Shukla,D.,(2014b):Area computation of internet traffic share problem with special reference to cyber crime environment, International Journal of Computer Networks and Wireless Communication, Vol. 4,No. 3,pp.208-219.

[20] Gangele,Sharad,Dongre,Ashish(2014c):Probability density estimation function of browser share curve for users web browser behaviour. International Journal of Engineering Research and Development, Vol. 10, Issue 6,pp.31-41.

[21] Gangele, Sharad, Dongre,Ashish (2014d): Two-call index based internet traffic sharing analysis in case of cyber crime environment of computer network, International Journal of Engineering Trend and Technology, Vol. 13, Issue 6,pp.271-280.

[22] Gangele, Sharad (2014): An Approach for Area Estimation towards Conjunction Control of Internet Traffic Sharing by Using Simpson 1/3ed Rule, International Journal of Engineering Trend and Technology, Vol. 16, Issue 2,pp.88-99.

[23] Shukla, D., Verma, Kapil and Gangele, Sharad (2015) :Approximating the Probability of Traffic Sharing by Numerical Analysis Techniques between Two Operators in a Computer Network, American Journal of Computer Science and Information Technology, Vol. 3, Issue 1,pp.26-39. 